\title{
Fabrication Process for Deep Submicron SQUID Circuits with Three Independent Niobium Layers
}

\author{
Silke Wolter ${ }^{1, *}$, Julian Linek ${ }^{2}$, Josepha Altmann ${ }^{3}{ }^{-}$, Thomas Weimann ${ }^{1}$, Sylke Bechstein ${ }^{3}$, Reinhold Kleiner ${ }^{2}$, \\ Jörn Beyer ${ }^{3}$, Dieter Koelle ${ }^{2}$ and Oliver Kieler ${ }^{1}$ \\ 1 Fachbereich Quantenelektronik, Physikalisch-Technische Bundesanstalt (PTB), \\ 38116 Braunschweig, Germany; thomas.weimann@ptb.de (T.W.); oliver.kieler@ptb.de (O.K.) \\ 2 Center for Quantum Science (CQ) and LISA+, Physikalisches Institut, Universität Tübingen, \\ 72076 Tübingen, Germany; julian.linek@uni-tuebingen.de (J.L.); kleiner@uni-tuebingen.de (R.K.); \\ koelle@uni-tuebingen.de (D.K.) \\ 3 Fachbereich Kryosensorik, Physikalisch-Technische Bundesanstalt (PTB), 10587 Berlin, Germany; \\ josepha.altmann@ptb.de (J.A.); sylke.bechstein@ptb.de (S.B.); joern.beyer@ptb.de (J.B.) \\ * Correspondence: silke.wolter@ptb.de; Tel.: +49-531-592-2465
}

Citation: Wolter, S.; Linek, J.; Altmann, J.; Weimann, T.; Bechstein, S.; Kleiner, R.; Beyer, J.; Koelle, D.; Kieler, O. Fabrication Process for Deep Submicron SQUID Circuits with Three Independent Niobium Layers. Micromachines 2021, 12, 350. https://doi.org/10.3390/mi12040350

Academic Editor: Antonio Vettoliere

Received: 22 February 2021

Accepted: 17 March 2021

Published: 24 March 2021

Publisher's Note: MDPI stays neutral with regard to jurisdictional claims in published maps and institutional affiliations.

Copyright: (c) 2021 by the authors. Licensee MDPI, Basel, Switzerland. This article is an open access article distributed under the terms and conditions of the Creative Commons Attribution (CC BY) license (https:/ / creativecommons.org/licenses/by/ $4.0 /)$.

\begin{abstract}
We present a fabrication technology for nanoscale superconducting quantum interference devices (SQUIDs) with overdamped superconductor-normal metal-superconductor (SNS) trilayer $\mathrm{Nb} / \mathrm{HfTi} / \mathrm{Nb}$ Josephson junctions. A combination of electron-beam lithography with chemicalmechanical polishing and magnetron sputtering on thermally oxidized Si wafers is used to produce direct current SQUIDs with 100-nm-lateral dimensions for $\mathrm{Nb}$ lines and junctions. We extended the process from originally two to three independent $\mathrm{Nb}$ layers. This extension offers the possibility to realize superconducting vias to all $\mathrm{Nb}$ layers without the HfTi barrier, and hence to increase the density and complexity of circuit structures. We present results on the yield of this process and measurements of SQUID characteristics.
\end{abstract}

Keywords: low-temperature superconductors; nanotechnology; SQUID; niobium technology

\section{Introduction}

Superconducting quantum interference devices (SQUIDs) are sensitive detectors of magnetic flux $\Phi$, used in a large variety of applications [1,2]. Strongly miniaturized direct current (dc) SQUIDs with lateral size in the $\mu \mathrm{m}$ range (microSQUIDs) or even sub- $\mu \mathrm{m}$ range (nanoSQUIDs) have received increasing attention during the last years [3,4], as they offer high spatial resolution and high sensitivity for the detection and investigation of magnetic sources on the nanoscale. Accordingly, nanoSQUIDs have promising applications for high-resolution scanning SQUID microscopy [5-13] and for the detection and investigation of individual magnetic nanoparticles (MNPs), nanowires or nanotubes [14-22]. Due to their small SQUID loop and size of the Josephson junctions (JJs) intersecting the loop, nanoSQUIDs are suited for operation in strong external magnetic fields [23,24]. In addition, downscaling the linewidth of the SQUID loop provides improved coupling to MNPs [25-27] as the magnetic field of which decays cubically with the distance. For a magnetic dipole placed in $10 \mathrm{~nm}$ distance to the SQUID loop, nanoscale SQUIDs can provide spin sensitivities even below $1 \mu_{\mathrm{B}} / \mathrm{Hz}^{1 / 2}$ ( $\mu_{\mathrm{B}}$ is the Bohr magneton) [7].

Most micro- and nanoSQUIDs are based on single layer devices with constriction-type JJs (Dayem bridges). The use of sandwich-type trilayer JJs, typically with $\mathrm{Nb}$ electrodes, is less common. However, this approach allows one to use a mature multilayer technology, which offers the realization of more complex and advanced nanoSQUID layouts, such as three-axis vector nanoSQUIDs [28] and auxiliary components, such as gradiometric feedback loops and transformers [29] with high yield and reproducibility of JJ parameters. The challenge that comes with this approach is the need for the fabrication of deep submicron 
JJs, to enable operation in strong magnetic fields. Accordingly, one needs to achieve JJs with high critical current density $j_{0}$, to provide large enough critical currents $I_{0}$. During the last years, it has been shown that the above-mentioned requirements can be met by fabricating devices based on trilayer JJs with superconducting (S) $\mathrm{Nb}$ electrodes and a normal conducting (N) HfTi barrier, using nanopatterning by electron beam lithography (EBL) and chemical-mechanical polishing (CMP) [30,31]. The critical current density of SNS JJs with HfTi barriers can be adjusted over a very wide range by the thickness of the $\mathrm{N}$ layer up to $j_{0} \approx 1 \mathrm{MA} / \mathrm{cm}^{2}$ at temperature $T=4.2 \mathrm{~K}$. Furthermore, lateral JJs sizes down to $80 \mathrm{~nm}$ diameter have been achieved [32,33]. We note that by the implementation of intrinsically shunted SNS JJs we avoid space-consuming external shunting resistors of the JJs in the circuit.

So far, our fabrication process has allowed for the realization of nanoSQUID designs with two independent $\mathrm{Nb}$ layers, i.e., $\mathrm{Nb}$ layers that are stacked on top of each other, separated by a highly insulating $\mathrm{SiO}_{2}$ layer, and that are patterned lithographically in separate steps. The two independent $\mathrm{Nb}$ layers can be connected vertically by SNS JJs with a HfTi barrier. For some applications, e.g., for the realization of nanoSQUID susceptometers, it is desirable to add at least one more independent $\mathrm{Nb}$ layer and to include vertical interconnections (vias) between the independent $\mathrm{Nb}$ layers, which are fully superconducting. There are already several $\mathrm{Nb}$-multilayer processes which offer high yield for up to ten superconducting layers with sub-micron vias (see, e.g., Refs. [34-37]). Those processes use a combination of ultraviolet-lithography with multiple CMP steps to planarize dielectric interlayers. Our fabrication technology, which is based on the combination of EBL and CMP, by contrast, offers the possibility to realize even smaller vias and JJs.

In this paper we describe the extension of our $\mathrm{Nb}$ multilayer technology for nanoSQUIDs from originally two to three independent layers of $\mathrm{Nb}$. This allows to significantly increase the density of circuit structures. Additionally, the possibility to realize direct vias between all independent $\mathrm{Nb}$ layers, i.e. directly connecting each $\mathrm{Nb}$ layer without the normal conducting HfTi barrier in-between (e.g., to realize purely superconducting bridges for coils), offers improved design flexibility. We present results on the yield of our extended fabrication process and the possibility to realize passive on-chip components with high aspect ratio like interdigital capacitors (IDCs), which allow for the realization of capacitances matching the designed value with high accuracy. Additionally, we demonstrate that the extension of our fabrication process does not influence the electric transport properties of our SQUIDs and can therefore be used to further develop more complex nanoSQUID circuits.

\section{Fabrication Technology}

The fabrication technology here employs EBL to ensure high alignment precision for nanopatterning of multilayer $\mathrm{Nb}$ structures. In addition, a CMP step is used to planarize the $\mathrm{SiO}_{2}$ layer between the first and second independent $\mathrm{Nb}$ layer. Both technologies are available at the clean room center at PTB Braunschweig and have been applied before in the fabrication of JJ-based circuits $[29,30,33]$. Figures $1-5$ illustrate the deposition and patterning steps (we note that the layer thicknesses are not to scale).

We use 3-inch $\mathrm{Si}$ wafer substrates with $300 \mathrm{~nm}$ thermally oxidized $\mathrm{SiO}_{2}$ (Figure 1). After a pre-cleaning step of the substrate (in-situ Ar sputter cleaning: $50 \mathrm{~W}$ for $2 \mathrm{~min}$ ), a $30 \mathrm{~nm} \mathrm{Al}{ }_{2} \mathrm{O}_{3}$ etch-stop layer is grown by magnetron sputtering deposition (MSD) in a high-vacuum chamber at room temperature. Subsequently the $\mathrm{Nb}-\mathrm{HfTi}-\mathrm{Nb}$ trilayer is grown by MSD. The trilayer consists of a $160 \mathrm{~nm} \mathrm{Nb}$ base layer, 20 to $22 \mathrm{~nm} \mathrm{Hf}_{\mathrm{wt} 50 \%} \mathrm{Ti}_{\mathrm{wt} 50 \%}$ (HfTi) and a $200 \mathrm{~nm} \mathrm{Nb}$ top layer for six wafers, which were fabricated for this paper. As mentioned above, the HfTi thickness can be adjusted, to obtain a desired critical current density, in a typical range from 17 to $26 \mathrm{~nm}$ [33]. To define the deep sub- $\mu \mathrm{m}$ JJs, a $30 \mathrm{~nm} \mathrm{Al}$ hard mask is patterned by lift-off, using EBL with PMMA as a positive resist. The $\mathrm{Nb}$ top layer is removed by inductively coupled plasma reactive ion etching (ICP RIE). To achieve optimum etching times, we use laser endpoint detection for ICP RIE of $\mathrm{Nb}$ and $\mathrm{SiO}_{2}$ layers. 
For etching the $\mathrm{Nb}$ top layer $\mathrm{SF}_{6}$ is used to obtain high etching rates and to ensure steep sidewalls approaching $90^{\circ}$. Ion beam etching (IBE) with Argon ions is applied to remove the HfTi barrier and simultaneously the Al hard mask.
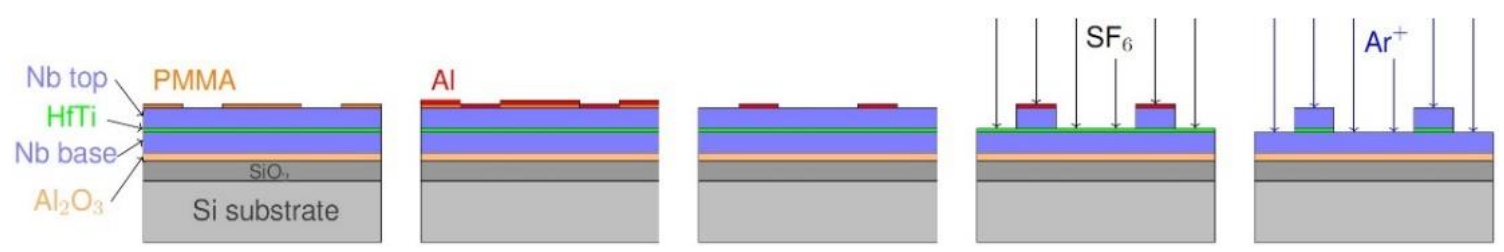

Figure 1. Layer scheme of the sub- $\mu \mathrm{m}$ range superconducting quantum interference device (nanoSQUID) fabrication process to define the Josephson junctions (JJs). After magnetron sputtering deposition (MSD) of the $\mathrm{Al}_{2} \mathrm{O}_{3}$ etch stop layer and the $\mathrm{Nb}-\mathrm{HfTi}-\mathrm{Nb}$ trilayer, the $\mathrm{Al}$ hard mask is patterned by a positive resist mask and a subsequent lift-off process and the $\mathrm{Nb}$ top layer of the trilayer is etched with inductively coupled plasma reactive ion etching (ICP RIE) $\mathrm{using} \mathrm{SF}_{6}$. Subsequently, the HfTi barrier and the Al mask are removed by Ar ion beam etching (IBE).

To pattern the $\mathrm{Nb}$ base layer (Figure 2) it is crucial for our optimized fabrication process to use the high-resolution negative resist ARN 7520.18 (and optimized EBL doses and proximity correction) which allows the patterning of the desired small SQUID geometry. ICP RIE is applied and the $\mathrm{Al}_{2} \mathrm{O}_{3}$ layer below the $\mathrm{Nb}$ base acts as a reliable etch stop. After removing the negative resist mask (using BAKER REZI-38), the wafer is covered by about $600 \mathrm{~nm} \mathrm{SiO}{ }_{2}$ by plasma enhanced chemical vapor deposition (PECVD) to not only ensure isolation between the first and the second independent $\mathrm{Nb}$ layer, but also to serve as a planar underlay for the following layers. Therefore, CMP is used to planarize the $\mathrm{SiO}_{2}$ layer to the height of the $\mathrm{Nb}$ top layer. This way $\mathrm{SiO}_{2}$ is removed from the $\mathrm{Nb}$ top layer which can be contacted electrically by the upper $\mathrm{Nb}$ layers afterwards.

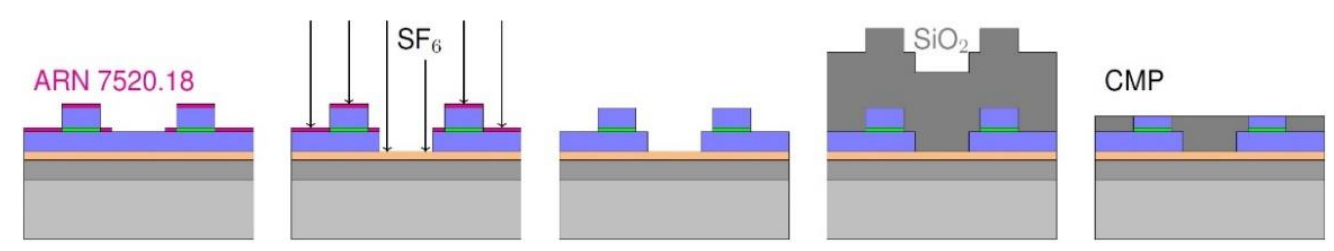

Figure 2. Layer scheme of the nanoSQUID fabrication process to pattern the $\mathrm{Nb}$ base layer and of the subsequent chemicalmechanical polishing (CMP) step. The $\mathrm{Nb}$ base layer is protected during the ICP RIE etching with $\mathrm{SF}_{6}$ by a high-resolution negative resist (ARN 7520.18) mask. Afterwards the mask is removed, and the wafer is covered with $\mathrm{SiO}_{2}$ via plasma enhanced chemical vapor deposition (PECVD). The wafer is planarized through the following CMP step.

In the next step (Figure 3), MSD (with in-situ Ar sputter cleaning: $50 \mathrm{~W}$ for $1 \mathrm{~min}$ ) is used to deposit the first $200 \mathrm{~nm}$ thick $\mathrm{Nb}$ wiring layer ( $\mathrm{Nb}$ wiring 1) which is again patterned by a mask of positive resist to define another Al hard mask. Equivalent to the patterning of the $\mathrm{Nb}$ top layer, the first $\mathrm{Nb}$ wiring layer is etched by $\mathrm{SF}_{6}$ with ICP RIE. Subsequently the $\mathrm{Al}$ hard mask is removed by a wet etching process using the developer maD-332 resp. the acid-based etching solution TechniEtch Al80.

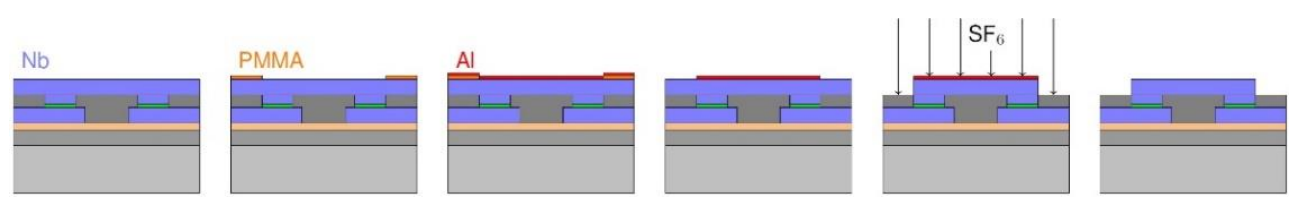

Figure 3. Layer scheme of the nanoSQUID fabrication process to define the first $\mathrm{Nb}$ wiring layer. After MSD of the $200 \mathrm{~nm}$ $\mathrm{Nb}$ layer, the $\mathrm{Al}$ hard mask is patterned by a positive resist mask and a subsequent lift-off process and the first $\mathrm{Nb}$ wiring layer is etched with ICP RIE using $\mathrm{SF}_{6}$. Subsequently, the $\mathrm{Al}$ mask is removed by using a wet etching step. 
In the next fabrication step, we use PECVD again to deposit another $300 \mathrm{~nm}$ thick layer of $\mathrm{SiO}_{2}$ on top of the wafer (Figure 4) to ensure the isolation between the first and second $\mathrm{Nb}$ wiring layers. We use a positive resist mask patterned by EBL and etching by ICP RIE with $\mathrm{CHF}_{3}$ to create small windows (vias) in the $\mathrm{SiO}_{2}$ layer. Here we can open contact windows down to the $\mathrm{Nb}$ base layer and to the first $\mathrm{Nb}$ wiring layer.
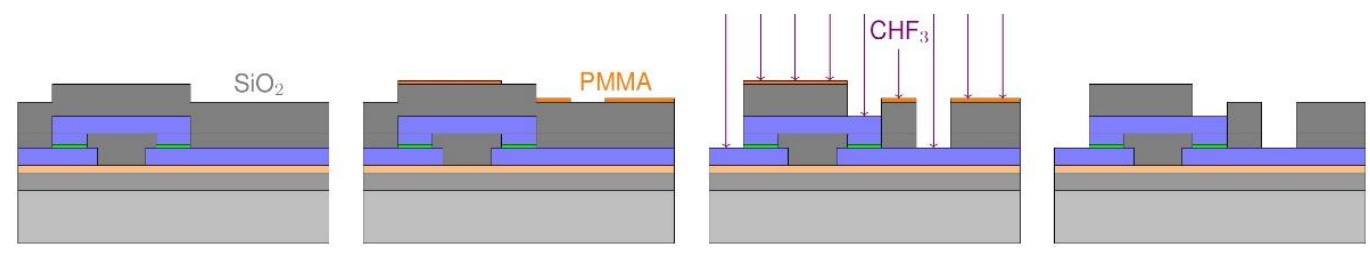

Figure 4. Layer scheme of the nanoSQUID fabrication process to etch windows (vias) into $\mathrm{SiO}_{2}$ to connect the $\mathrm{Nb}$ layers. $\mathrm{SiO}_{2}$ is deposed on top of the structure via PECVD and protected by a patterned positive resist mask during ICP RIE with $\mathrm{CHF}_{3}$.

As all $\mathrm{Nb}$ layers, $\mathrm{Nb}$ wiring 2 is deposited starting with an in-situ Ar sputter cleaning (50 W for $1 \mathrm{~min}$ ) by MSD (Figure 5). The thickness of the second $\mathrm{Nb}$ wiring layer must be comparably large $(800 \mathrm{~nm})$ compared to the underlying layers to ensure that the superconducting $\mathrm{Nb}$ layer does not break off at the edges, because of the uneven structures when connecting the $\mathrm{Nb}$ base layer. For the last $\mathrm{Nb}$ etching step, another $\mathrm{Al}$ hard mask, which is again formed by an EBL patterned positive resist mask and a lift-off process, is needed. By ICP RIE with $\mathrm{SF}_{6}$ the second $\mathrm{Nb}$ wiring layer is patterned.
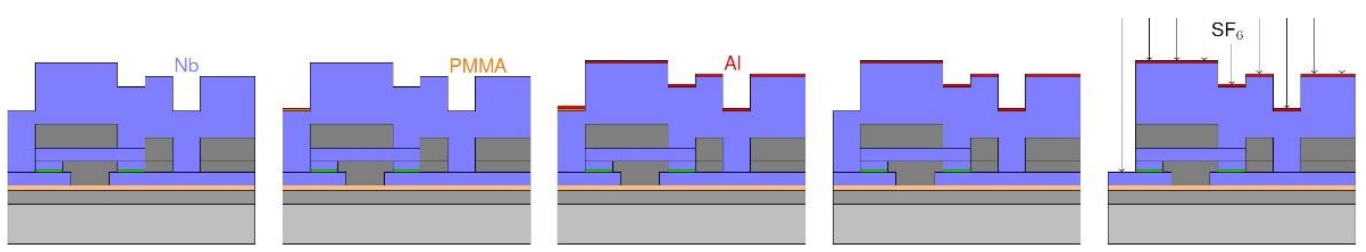

Figure 5. Layer scheme of the nanoSQUID fabrication process to define the second $\mathrm{Nb}$ wiring layer. After MSD of this third independent $\mathrm{Nb}$ layer the $\mathrm{Al}$ hard mask is patterned by a positive resist mask and a subsequent lift-off process and the second $\mathrm{Nb}$ wiring layer is etched with ICP RIE using $\mathrm{SF}_{6}$.

We note that an AuPd resistive layer can be integrated between the first and second $\mathrm{Nb}$ wiring layer fabrication step (not shown here) to form passive components such as resistors. By choosing the thickness of the AuPd layer, a desired sheet resistance can be adjusted; for example, a $70 \mathrm{~nm}$ AuPd layer results in a sheet resistance of $5 \Omega$.

In the following sections, we present measurement results concerning the yield and the functionality of parallel-plate capacitors to test the isolation between independent $\mathrm{Nb}$ layers and of vias to examine vertical connections between the three independent $\mathrm{Nb}$ layers. Furthermore, we investigated the realization of large fine pitch interdigital capacitors. Additionally, SQUIDs and single JJs were fabricated and investigated regarding their electrical transport characteristics. Six wafers were fabricated during the optimization of the presented process with slightly different process parameters like HfTi thicknesses and etching times.

\section{Evaluation of Isolation and via Yield}

In order to examine the quality of isolation and vias between $\mathrm{Nb}$ layers, we fabricated and characterized parallel-plate capacitor structures with varied dimensions, $10 \mu \mathrm{m} \times 10 \mu \mathrm{m}$ to $800 \mu \mathrm{m} \times 800 \mu \mathrm{m}$, between all independent layers of $\mathrm{Nb}$ (base, wiring 1, wiring 2) and additionally between the trilayer and the second wiring layer. In Figure 6 the different types of capacitors are sketched. 


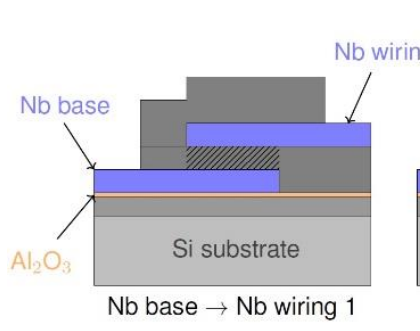

(a)

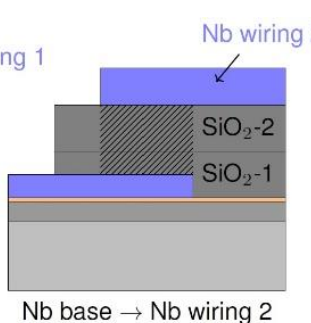

(b)

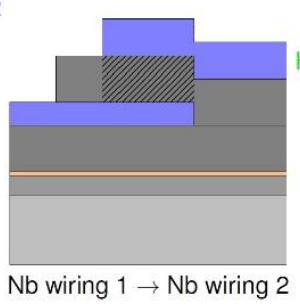

(c)

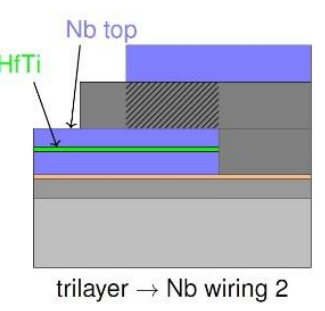

(d)

Figure 6. Layer scheme of the different types of parallel-plate capacitors. The plates of capacitors are in all three independent layers of $\mathrm{Nb}(\mathbf{a}-\mathbf{c})$ as well as in the trilayer $(\mathbf{d})$, and they are separated by $\mathrm{SiO}_{2}$ as the dielectric layer. The hatched area represents the volume between the plates.

First, we measured the resistance of the parallel-plate capacitors at room temperature with a wafer prober system (SÜSS MicroTec PM5 [38]) using a Keithley Model 2000 multimeter ${ }^{1}$. Thereby, we refer to the electrical contact between the three independent $\mathrm{Nb}$ layers as "open", i.e., there are no unintentional shorts, if the measured resistance is larger than $120 \mathrm{M} \Omega$. The results are summarized in Table 1. Furthermore, we note that we refer to a "high" yield even though it is less than $100 \%$, since we fabricate devices for research purposes, not for industrial purposes, and the investigated less complex SQUIDs showed functionality (see Section 5).

Those measurements showed a yield of $70 \%$ for a total of 443 investigated capacitors with different sizes and plates in $\mathrm{Nb}$ base and $\mathrm{Nb}$ wiring 1 . For capacitors with plates in $\mathrm{Nb}$ base and $\mathrm{Nb}$ wiring 2, and for capacitors between the two wiring layers, a very high yield of $97 \%$ for 238 and $91 \%$ for 241 investigated capacitors was achieved, respectively. Capacitors with plates in the trilayer and $\mathrm{Nb}$ wiring 2 showed a high yield of $85 \%$ for a total of 47 investigated capacitors. The larger capacitors between the $\mathrm{Nb}$ base layer and the first wiring layer stemming from the middle of all wafers showed a slightly lower yield. We attribute this result to the inhomogeneity of our CMP step in which the center part of the wafer is somewhat stronger polished than the edges. This unevenness is likely to increase the occurrence of shorts through the insulation layer. Moreover, for some wafers the yield of capacitors between the two wiring layers was quite low. In those cases, clearly visible Niobium residuals ("fences") appeared due to gaps between the first wiring layer and the dielectric and led to shorts between the wiring layers.

Table 1. Total number of investigated capacitors between different $\mathrm{Nb}$ layers for 6 different wafers and yield for each type of capacitor (corresponding to Figure 6) without shorts.

\begin{tabular}{|c|c|c|c|c|c|c|}
\hline Capacitors & Wafer 1 & Wafer 2 & Wafer 3 & Wafer 4 & Wafer 5 & Wafer 6 \\
\hline $\begin{array}{c}\text { (a) Base } \\
\rightarrow \text { Wiring } 1\end{array}$ & $\begin{array}{c}205 \\
(\mathbf{7 1} \%)\end{array}$ & $\begin{array}{c}200 \\
(73 \%)\end{array}$ & $\begin{array}{c}10 \\
(50 \%)\end{array}$ & $\begin{array}{c}10 \\
(30 \%)\end{array}$ & $\begin{array}{c}9 \\
(67 \%)\end{array}$ & $\begin{array}{c}9 \\
(56 \%)\end{array}$ \\
\hline $\begin{aligned} & \text { (b) Base } \\
& \rightarrow \text { Wiring } 2\end{aligned}$ & $\begin{array}{c}100 \\
(\mathbf{1 0 0} \%)\end{array}$ & $\begin{array}{c}100 \\
(\mathbf{9 7 \%})\end{array}$ & $\begin{array}{c}10 \\
(\mathbf{9 0 \%})\end{array}$ & $\begin{array}{c}10 \\
(\mathbf{8 0} \%)\end{array}$ & $\begin{array}{c}9 \\
(89 \%)\end{array}$ & $\begin{array}{c}9 \\
(100 \%)\end{array}$ \\
\hline $\begin{array}{l}\text { (c) Wiring } 1 \\
\rightarrow \text { Wiring } 2\end{array}$ & $\begin{array}{c}100 \\
(\mathbf{1 0 0 \%})\end{array}$ & $\begin{array}{c}103 \\
(\mathbf{1 0 0 \%})\end{array}$ & $\begin{array}{c}10 \\
(70 \%)\end{array}$ & $\begin{array}{c}10 \\
(\mathbf{0} \%)\end{array}$ & $\begin{array}{c}9 \\
(22 \%)\end{array}$ & $\begin{array}{c}9 \\
(78 \%)\end{array}$ \\
\hline $\begin{array}{l}\text { (d) Trilayer } \\
\rightarrow \text { Wiring } 2\end{array}$ & $\begin{array}{c}9 \\
(\mathbf{1 0 0} \%)\end{array}$ & - & $\begin{array}{c}10 \\
(80 \%)\end{array}$ & $\begin{array}{c}10 \\
(80 \%)\end{array}$ & $\begin{array}{c}9 \\
(\mathbf{1 0 0} \%)\end{array}$ & $\begin{array}{c}9 \\
(67 \%)\end{array}$ \\
\hline
\end{tabular}

The capacitance $C$ of selected parallel-plate capacitor structures from wafers 5 und 6 was measured at $4.2 \mathrm{~K}$ using a KEYSIGHT E4980A LCR-meter [38]. This was done to test the low-temperature functionality of the capacitors as well as to obtain an estimate of the thickness $d$ of the two $\mathrm{SiO}_{2}$ layers $\left(\mathrm{SiO}_{2}-1\right.$ and $\left.\mathrm{SiO}_{2}-2\right)$. To obtain $d$, the data were fitted according to

$$
C=\varepsilon_{\mathrm{r}} \varepsilon_{0} A / d+\text { offset }
$$


where $\varepsilon_{0}$ is the dielectric constant of vacuum and $\varepsilon_{\mathrm{r}}=4$ [39] is the permittivity of our PECVD SiO ${ }_{2}$. Since $C(A=0)=0$ should be valid, we correct the measured capacitances by a constant offset, $(33.6 \pm 2.4) \mathrm{pF}$, which is assumed to be independent of the size of the capacitors and can be attributed to the parasitic capacitance of the measurement setup. We note that the offset partially results from so called "fringing fields" [40], whereas the effect is stronger for the smaller capacitors and therefore, the offset is strictly spoken not a constant value. However, since the deviation though the effect of the fringing fields for our capacitors is not notable (only about $1.3 \%$ of the capacitance for the smallest capacitors) compared to the measurement error, we treat the offset like a constant here. The corrected values and the linear fit functions (Equation (1) for offset $=0$ ) are shown in Figure 7 for all four types of capacitors as a function of the area $A$ of the parallel plates of the capacitors.

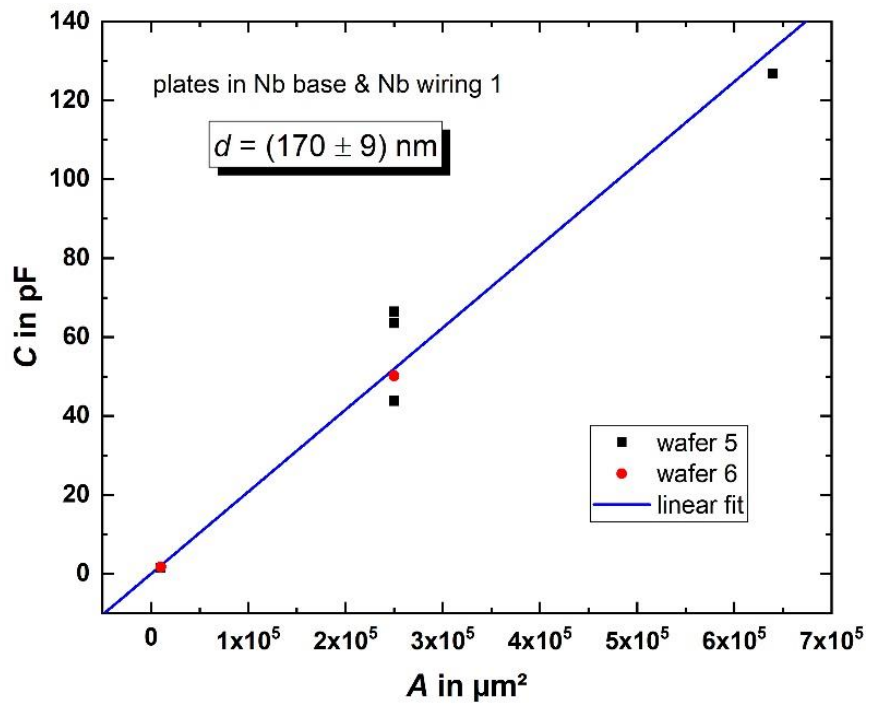

(a)

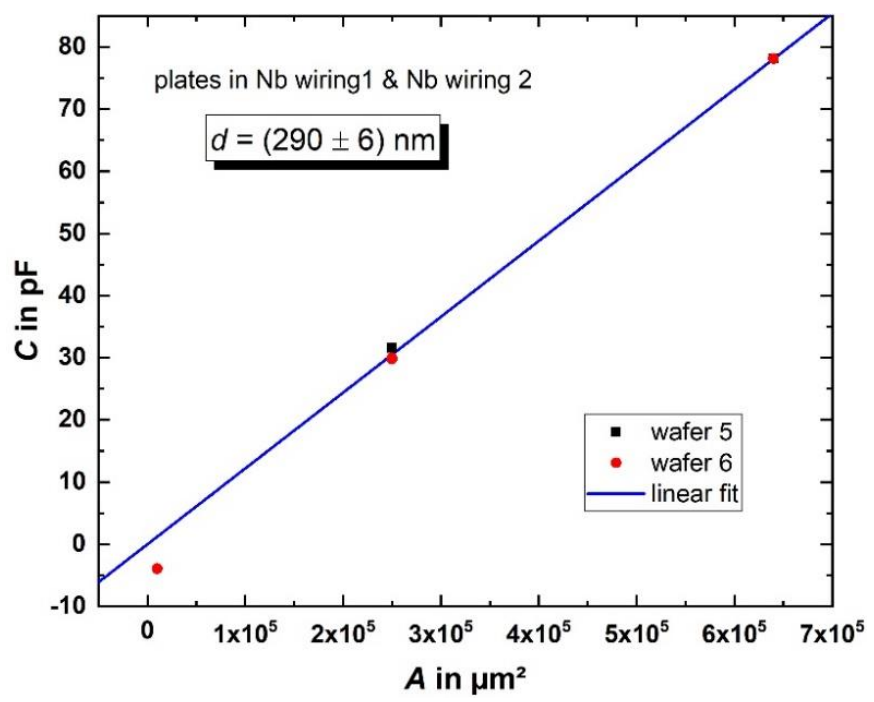

(c)

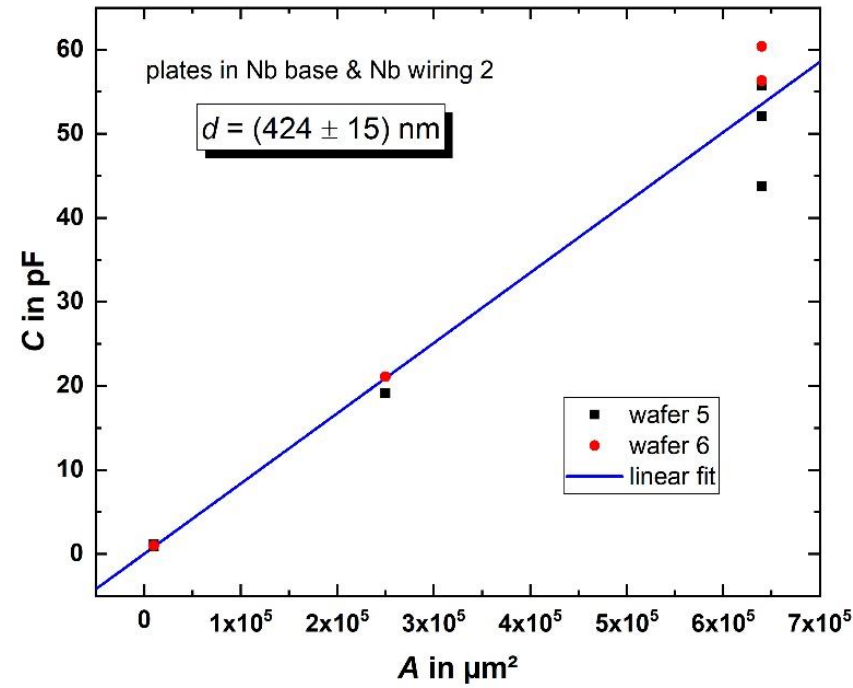

(b)

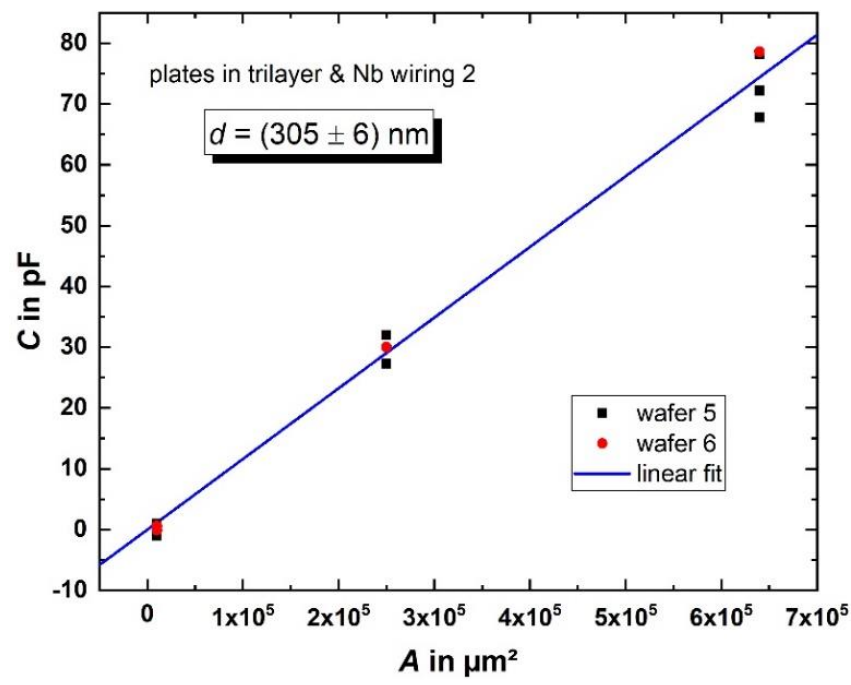

(d)

Figure 7. Measured capacitances (corrected values) of capacitors (corresponding to Figure 6) on wafers 5 and 6 with different sizes $(100 \mu \mathrm{m} \times 100 \mu \mathrm{m} \ldots 800 \mu \mathrm{m} \times 800 \mu \mathrm{m})$. The thickness of the dielectric layers was calculated according to Equation (1) from the fit parameters for capacitors with plates in the $\mathrm{Nb}$ base and first wiring layer (a), in the $\mathrm{Nb}$ base and second wiring layer (b), in the two wiring layers (c) and in the trilayer and second wiring layer (d).

The effective $\mathrm{SiO}_{2}$ thickness between the different capacitors with plates in the $\mathrm{Nb}$ base layer and the first $\mathrm{Nb}$ wiring layer (Figure 7a) is $d=(170 \pm 9) \mathrm{nm}$. For capacitors 
with plates in the $\mathrm{Nb}$ base and second wiring layer (Figure $7 \mathrm{~b}$ ), an effective thickness of $d=(424 \pm 15) \mathrm{nm} \mathrm{SiO} 2$ between the two plates is estimated. Since the $\mathrm{SiO}_{2}-2$ layer has a nominal thickness of $300 \mathrm{~nm}$, there is a significant mismatch between the estimated thickness of the $\mathrm{SiO}_{2}-1$ layer from the measurement shown in Figure $7 \mathrm{a}$ and the estimation of the thickness of the $\mathrm{SiO}_{2}-1$ layer from the measurement shown in Figure $7 \mathrm{~b}$, reduced by the nominal thickness of the $\mathrm{SiO}_{2}-2$ layer. The inter-wafer inhomogeneity of the CMP process causes the thickness of the $\mathrm{SiO}_{2}-1$ layer of wafer 5 and wafer 6 to be less predictable. Additionally, the intra-wafer inhomogeneity of the CMP process depends on the structure dimensions and density, causing the thickness of the remaining $\mathrm{SiO}_{2}-1$ layer to differ across each wafer. Both effects can be observed in Figure $7 \mathrm{~b}$, where the capacitances are larger for wafer 6 than for wafer 5 due to stronger polishing, and the spread of the measured capacitances is stronger for larger structures.

For capacitors with plates in both wiring layers (Figure 7c) and for capacitors with plates in the trilayer and the second wiring layer (Figure 7d), we obtain $d=(290 \pm 6)$ and (305 \pm ) $\mathrm{nm}$, which is $97 \%$ and $101 \%$, respectively, of the intended thickness of the $\mathrm{SiO}_{2}-2$ layer. The differences to the desired value are within the accuracy of the measurement. Since no CMP step was used for those capacitors, the thickness of the deposited $\mathrm{SiO}_{2}-2$ layer could be adjusted precisely and therefore, the capacitances match the designed values accurately.

Altogether, the fabricated capacitor structures show no drastic nonlinear dependence of the capacitance on the area of the parallel plates. The thickness of the dielectric $\mathrm{SiO}_{2}-2$ layer could be estimated within the scope of measurement accuracy and matches the expectations. The estimation of the thickness of the $\mathrm{SiO}_{2}-1$ layer shows a larger uncertainty due to the inhomogeneity of the CMP process, but not the area of the capacitors' plates which can be fabricated with a very high accuracy.

In addition to examining the quality of the isolation between the $\mathrm{Nb}$ layers, we studied the yield characteristics of direct vias connecting the three independent layers of $\mathrm{Nb}$ as well as of JJs of varied size. The direct connections from $\mathrm{Nb}$ wiring 2 to $\mathrm{Nb}$ wiring 1 and to the $\mathrm{Nb}$ base layer avoid contact through the normal conducting barrier layer (Figure 8a,b). These superconducting vias were designed to vary in size from $1 \mu \mathrm{m} \times 1 \mu \mathrm{m}$ to $10 \mu \mathrm{m} \times 10 \mu \mathrm{m}$. The JJs, or "JJ vias" (contacts opened by CMP), with lateral dimensions $0.1 \mu \mathrm{m} \times 0.1 \mu \mathrm{m}$ to $5 \mu \mathrm{m} \times 5 \mu \mathrm{m}$, naturally include the HfTi barrier (Figure 8c).

To investigate the yield of functional vias ( $\mathrm{Nb}$ vias, connecting $\mathrm{Nb}$ wiring 2 with $\mathrm{Nb}$ base or $\mathrm{Nb}$ wiring 1 , and $\mathrm{JJ}$ vias, connecting $\mathrm{Nb}$ wiring 1 with $\mathrm{Nb}$ base), we measured the resistance of the vias including their on-chip leads at room temperature with a wafer prober system. The expected resistance is $R \approx 90 \Omega$, which corresponds to the resistance of the on-chip leads, calculated from the sheet resistance of niobium. The HfTi barrier is expected to result in an additional resistance of about $20 \Omega$. Therefore, we define a via as "functional" if the measured resistance does not exceed the expected total resistance of the on-chip leads and the HfTi barrier by more than $25 \%$ (i.e., $R \approx 140 \Omega$ ). The total number and the yield of functional vias is presented in Table 2.

Table 2. Total number of investigated vias for 6 different wafers (corresponding to Figure 8) and yield for each type of via.

\begin{tabular}{|c|c|c|c|c|c|c|}
\hline Capacitors & Wafer 1 & Wafer 2 & Wafer 3 & Wafer 4 & Wafer 5 & Wafer 6 \\
\hline $\begin{aligned} & \text { (a) Base } \\
& \rightarrow \text { Wiring } 2\end{aligned}$ & $\begin{array}{c}104 \\
(93 \%)\end{array}$ & $\begin{array}{c}100 \\
(\mathbf{8 0} \%)\end{array}$ & $\begin{array}{c}10 \\
(80 \%)\end{array}$ & $\begin{array}{c}10 \\
(\mathbf{1 0 0} \%)\end{array}$ & $\begin{array}{c}9 \\
(\mathbf{1 0 0} \%)\end{array}$ & $\begin{array}{c}9 \\
(89 \%)\end{array}$ \\
\hline $\begin{array}{l}\text { (b) Wiring } 1 \\
\rightarrow \text { Wiring } 2\end{array}$ & $\begin{array}{c}102 \\
(95 \%)\end{array}$ & $\begin{array}{c}100 \\
(\mathbf{9 9} \%)\end{array}$ & $\begin{array}{c}10 \\
(\mathbf{1 0 0} \%)\end{array}$ & $\begin{array}{c}10 \\
(\mathbf{1 0 0} \%)\end{array}$ & $\begin{array}{c}9 \\
(\mathbf{1 0 0} \%)\end{array}$ & $\begin{array}{c}9 \\
(44 \%)\end{array}$ \\
\hline $\begin{aligned} & \text { (c) Trilayer } \\
\rightarrow & \text { Wiring } 1 \text { (JJ vias) }\end{aligned}$ & $\begin{array}{c}21 \\
(90 \%)\end{array}$ & $\begin{array}{c}21 \\
(\mathbf{8 1} \%)\end{array}$ & $\begin{array}{c}22 \\
(\mathbf{1 8 \%})\end{array}$ & $\begin{array}{c}22 \\
(\mathbf{1 8 \%})\end{array}$ & $\begin{array}{c}21 \\
(\mathbf{1 0} \%)\end{array}$ & $\begin{array}{c}21 \\
(\mathbf{1 0} \%)\end{array}$ \\
\hline
\end{tabular}

A yield of $88 \%$ for a total of 242 vias in different sizes connecting the $\mathrm{Nb}$ base layer and the second wiring layer was achieved. Vias connecting the two wiring layers got a 
high yield of $95 \%$ for a total of 240 measured vias with different sizes. The investigation of the JJ vias showed a yield of $38 \%$ for 128 structures.

To assess the functional quality of the $\mathrm{Nb}$ vias also at low temperatures, transport current tests were performed at $4.2 \mathrm{~K}$ on one exemplary $\mathrm{Nb}$ via, connecting the $\mathrm{Nb}$ base layer and $\mathrm{Nb}$ wiring 2, with lateral size of $2 \mu \mathrm{m} \times 2 \mu \mathrm{m}$ from wafer 2 . A bias current up to $100 \mathrm{~mA}$, corresponding to a current density of up to $2.5 \times 10^{6} \mathrm{~A} / \mathrm{cm}^{2}$, could be applied without a measurable voltage drop. This demonstrates the expected significant increase in critical current density for direct $\mathrm{Nb}$ vias compared to JJ vias. We note that all vias (superconductor-superconductor and superconductor-normal metal-superconductor vias), which were functional at room temperature and which were investigated at low temperature (including vias which are parts of devices) showed superconducting properties.

As discussed in Section 2, there is only one $\mathrm{SiO}_{2}$ plasma etching step within the fabrication process (see Figure 4). Note, that for contacting from the $\mathrm{Nb}$ wiring 2 layer to the $\mathrm{Nb}$ wiring 1 layer, only the $\mathrm{SiO}_{2}-2$ layer must be removed whereas for contacting to the $\mathrm{Nb}$ base layer, the $\mathrm{SiO}_{2}-2$ layer as well as the $\mathrm{SiO}_{2}-1$ layer must be etched. As a consequence, an appropriate etching time need to be applied to open windows both to the $\mathrm{Nb}$ base layer as well as to the $\mathrm{Nb}$ wiring 1 layer. Furthermore, the thickness of the $\mathrm{SiO}_{2}-1$ layer is inhomogeneous across the wafers due to CMP. This situation makes it difficult to find an optimal etching time. Hence, we observe a smaller yield for $\mathrm{Nb}$ base to $\mathrm{Nb}$ wiring 2 connections for some wafers, where the etching time was not sufficient to open all vias down to the $\mathrm{Nb}$ base layer. For the vias connecting the two wiring layers, a yield of $95 \%$ was determined. However, since we observed so-called $\mathrm{Nb}$ fences here, the apparently high yield could be caused to a certain extent by unintentional shorts between the $\mathrm{Nb}$ layers. The yield of JJ vias is high for the first two wafers, but a higher inhomogeneity of CMP for the wafers 3 to 6 lead to a significant decrease in functional JJ vias.

In summary, the overall yield of parallel-plate capacitors and $\mathrm{Nb}$ vias is very high ( $83 \%$ for all capacitors and $91 \%$ for all $\mathrm{Nb}$ vias) and might be increased in the future by optimizing etching times or, e.g., by introducing an additional CMP step. In general, the laser endpoint detection for etching $\mathrm{SiO}_{2}$ and $\mathrm{Nb}$ is extremely important for the fabrication process. However, the optimum etching time is difficult to find due to inhomogeneous layer thicknesses and etching rates.

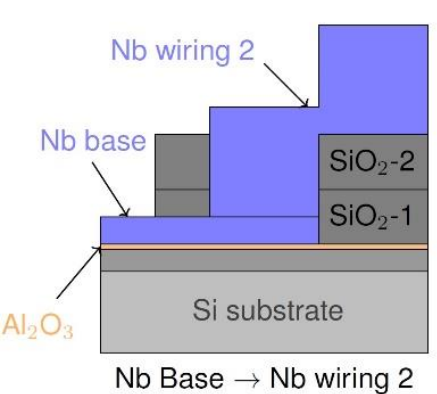

(a)

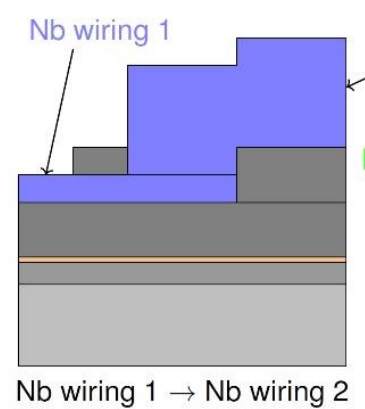

(b)

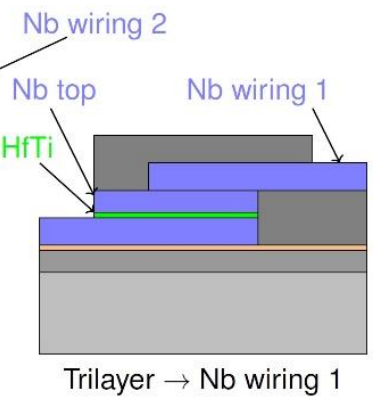

(c)

Figure 8. Layer scheme of the different types of vias connecting three independent layers of $\mathrm{Nb}$. The connections from the second $\mathrm{Nb}$ wiring layer to the $\mathrm{Nb}$ base (a) and first $\mathrm{Nb}$ wiring layer (b) are fully superconducting; the connection between $\mathrm{Nb}$ wiring 1 and $\mathrm{Nb}$ base (c) is disrupted by a normal conducting HfTi barrier.

\section{Characterization of Structures with High Aspect Ratio}

The process described above was also used to realize fine pitch IDCs in the Nb base layer with extremely high aspect ratio $A R=n \times L / p$. Here, $n \times L$ is the total length of the IDC, i.e., the total number of fingers on both sides of the IDC $(n)$ times the length $L$ of one finger. The pitch $p$ is defined as the distance between the centers of two neighboring fingers, i.e., $p$ is the sum of the linewidth of one finger and the gap between two fingers. 
We fabricated IDCs with $L=4183 \mu \mathrm{m}, n=500,750$, and 1000 and with $p=1.4,2$, and $4 \mu \mathrm{m}$ for different IDCs with the same $n$.

An AR of up to $2.2 \times 10^{6}$ (with $n=750$ and $p=1.4 \mu \mathrm{m}$ ) was achieved. Figure 9 shows scanning electron microscopy (SEM) images of a fabricated IDC, indicating that large structures with small linewidths can be fabricated without any visible defects. The capacitance of the IDCs was measured in liquid He at $T=4.2 \mathrm{~K}$ with an LCR-meter. To estimate $C$ from the geometry of the IDCs, we used the relation ([41] p. 96, Equation (4.47a)):

$$
C[\mathrm{pF}]=3.937 \cdot 10^{-5} \cdot L\left(\varepsilon_{r}+1\right) \cdot\{0.11(n-3)+0.252\} \text { for } L \text { in } \mu \mathrm{m},
$$

with $n=\{500,750,1000\}$ and $L=4183 \mu \mathrm{m}$, which is valid for IDCs with linewidth = gap such as the IDCs presented here. For the effective dielectric constant of the structures, $\varepsilon_{r} \approx 10$ was estimated in advance for the layout, as the value of the Si-substrate $\varepsilon_{r, S i} \approx 12$ is slightly reduced by the smaller value $\varepsilon_{r, S i O 2} \approx 4$ [39] of the insulating $\mathrm{SiO}_{2}$ on top of the IDCs and between the fingers.

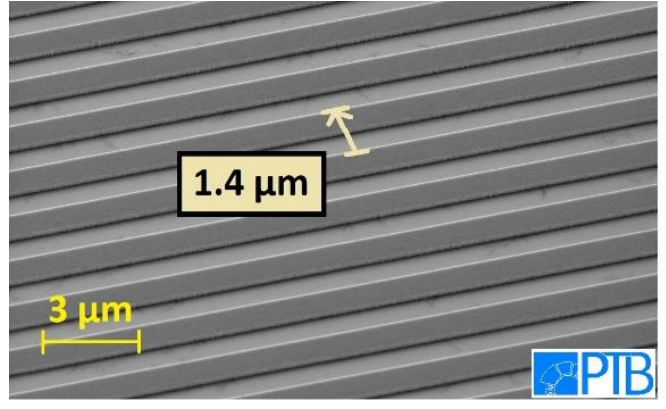

(a)

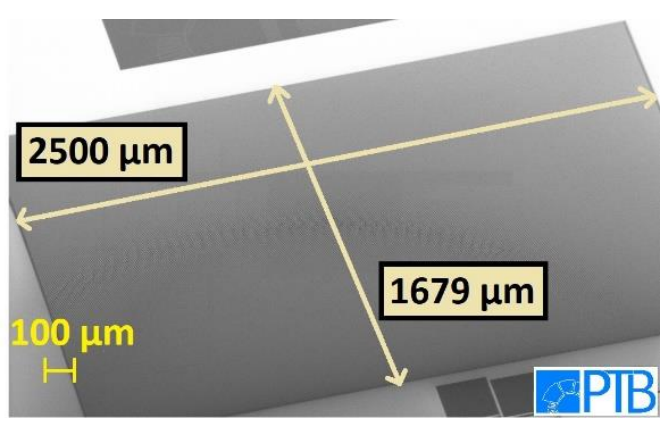

(b)

Figure 9. Scanning electron microscopy (SEM) images of a fine pitch interdigital capacitors (IDC) fabricated in the $\mathrm{Nb}$ base layer. (a) The IDC's pitch $=1.4 \mu \mathrm{m}$ equals the design value. (b) The large IDC shown here exhibits no visible defects.

The measured capacitances, reduced by an offset $(33.6 \mathrm{pF})$, which is attributed to the parasitic capacitances of the measuring system, are shown in Figure 10. We used a linear fit function according to Equation (2) to derive the effective dielectric constant $\varepsilon_{r, e f f}=(9.89 \pm 0.01)$, which deviates only $1 \%$ from the value, which was estimated for the layout beforehand. Regarding the capacitances, for IDCs with $n=500$, we expected $100 \mathrm{pF}$ per design. The mean value of the measured capacitances for these IDCs, having different pitches $(1.4 \mu \mathrm{m}, 2 \mu \mathrm{m}$, and $4 \mu \mathrm{m})$, was (97 $\mathrm{pF} \pm 3) \mathrm{pF}$.

Furthermore, we define an IDC as "functional", if there are no unintentional shorts, i.e., the measured resistance is larger than $120 \mathrm{M} \Omega$. The yield of functional IDCs was found to be $\approx 14 \%$. We attribute this comparably low yield to the large area of the structure that is vulnerable to small particles masking the $\mathrm{Nb}$ between the fingers during the etching step. Nevertheless, the results demonstrate functional IDCs with capacitance values matching the designed values with high accuracy. 


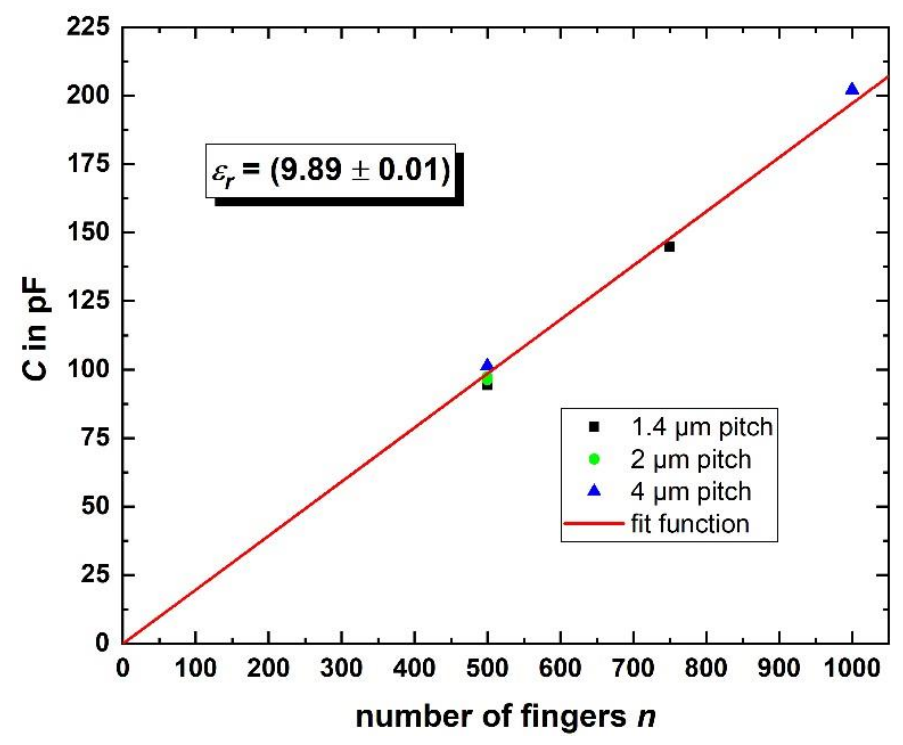

Figure 10. Measured capacitance of the investigated IDCs with different pitches. With a linear fit function according to Equation (2), the effective dielectric constant of the capacitors' dielectric can be estimated.

\section{Determination of Basic Electric Transport Properties of SQUID Test Structures Based on Submicron Josephson Junctions}

To demonstrate the suitability of the presented fabrication process for the realization of nanoSQUIDs we designed [42], fabricated, and characterized dc SQUID gradiometers with SNS JJs with nominal lateral JJ size of $200 \mathrm{~nm} \times 200 \mathrm{~nm}$. A closed superconducting loop is constructed in the $\mathrm{Nb}$ base layer and is connected via two JJs to the modulation line in the first $\mathrm{Nb}$ wiring layer. This results in a parallel gradiometer. The SQUID inductance $L_{S Q}$ is then given by one half of the inductance of one loop of the gradiometer. The magnetic flux $\Phi$ in the SQUID can be adjusted by the modulation current $I_{\text {mod }}$ through the modulation line. Since the $\mathrm{Nb}$ wiring 2 layer is not needed for this SQUID design, the SQUIDs are covered by the $\mathrm{SiO}_{2}-2$ layer. We show that the extension of our process from originally two to three independent layers of $\mathrm{Nb}$ does not downgrade the electric transport properties of our fabricated devices.

The SQUID loop of the first type of SQUID gradiometer (SQ-1 in Figure 11a) was designed to be $10 \mu \mathrm{m} \times 10 \mu \mathrm{m}$ large and to have a linewidth of $4.6 \mu \mathrm{m}$. A second type of SQUID gradiometer (SQ-2 in Figure 11b) was fabricated with a much smaller $1.5 \mu \mathrm{m} \times 1.5 \mu \mathrm{m}$ outer loop size with significantly reduced linewidth of $250 \mathrm{~nm}$. In addition to the SQUIDs, single SNS JJs with the same nominal area of $0.04 \mu \mathrm{m}^{2}$ were fabricated to investigate their electric transport characteristics. All data shown below were taken at liquid He temperature $(T=4.2 \mathrm{~K})$.

The current-voltage characteristics (IVCs), with $I_{\text {mod }}$ adjusted to obtain maximum critical current $I_{\mathcal{C}, \max }$ and minimum critical current $I_{c, \min }$, are shown in Figure 12a for an exemplary SQUID of the type SQ-1 with JJs having a barrier thickness of $d_{\text {HfTi }}=20 \mathrm{~nm}$ and in Figure 12b for another exemplary SQUID (type SQ-2) with a slightly thicker HfTi barrier $\left(d_{\mathrm{HfTi}}=21 \mathrm{~nm}\right)$. The IVCs with maximum critical current can be well described within the resistively and capacitively shunted junction (RCSJ) model [43,44], with negligible capacitance and with negligible noise rounding, which is consistent with the small noise parameter $\Gamma=2 \pi k_{B} T /\left(I_{0} \Phi_{0}\right) \approx 2 \times 10^{-3}$ (with the Boltzmann constant $k_{B}$ and average single JJ critical current $\left.I_{0}=I_{c, \max } / 2\right)$. The junctions exhibit a critical current density $j_{c} \approx 298 \mathrm{kA} / \mathrm{cm}^{2}$ and $j_{c} \approx 216 \mathrm{kA} / \mathrm{cm}^{2}$ for SQ-1 and SQ-2, respectively. They have a normal resistance $R_{S Q} \approx 343 \mathrm{~m} \Omega$ (SQ-1) up to $415 \mathrm{~m} \Omega$ (SQ-2), leading to characteristic voltages in the range of $V_{c}=I_{c, \max } R_{S Q} \approx 82 \mu \mathrm{V}$ (SQ-1) resp. $72 \mu \mathrm{V}$ (SQ-2). 


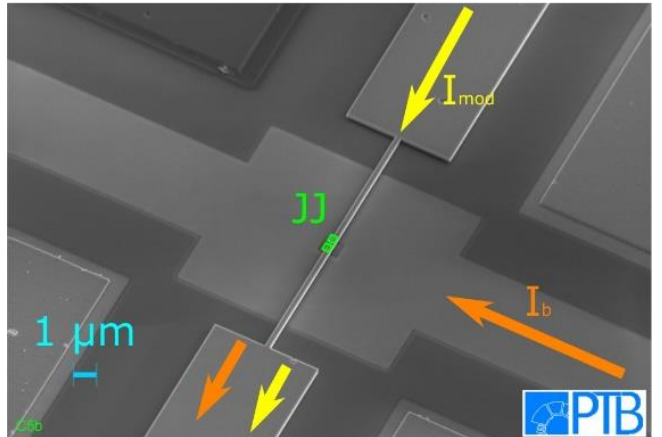

(a)

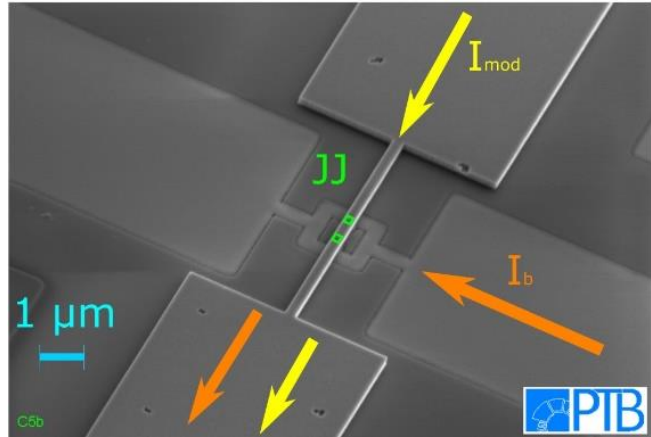

(b)

Figure 11. SEM images of both types of fabricated dc SQUID parallel gradiometers, (a) SQ-1 and (b) SQ-2 after the fabrication of the $\mathrm{Nb}$ wiring 1 layer. A closed superconducting loop in the $\mathrm{Nb}$ base layer is connected via two JJs to a modulation line in the $\mathrm{Nb}$ wiring 1 layer. The bias current $I_{b}$, and the modulation current $I_{\text {mod }}$ are indicated by arrows and the positions of the JJs are indicated by squares. The voltage across the SQUID is measured between the bias current terminals.

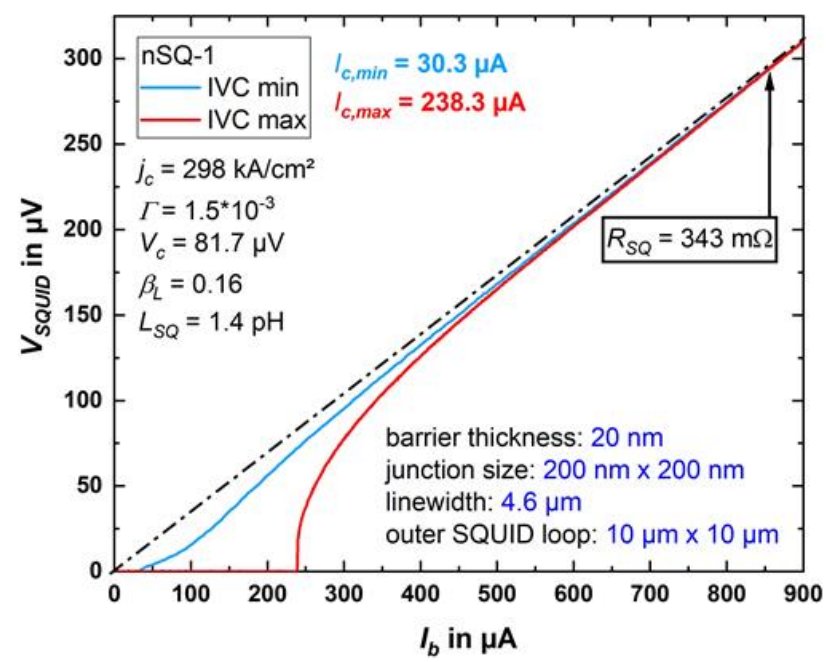

(a)

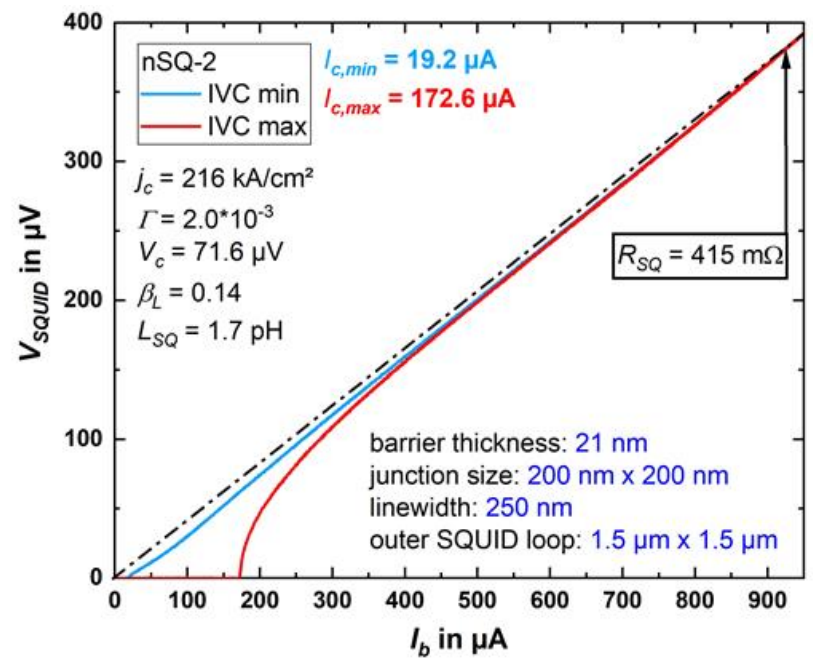

(b)

Figure 12. The current-voltage characteristics (IVCs) at $4.2 \mathrm{~K}$ of SQ-1 (a) and SQ-2 (b), with $I_{\text {mod }}$ adjusted to achieve maximum (IVC max) and minimum (IVC min) critical current. The characteristic electric transport and geometric parameters of the SQUIDs are indicated.

For SQ-2, we show in Figure 13a the critical current oscillations $I_{c}\left(I_{\text {mod }}\right)$; Figure $13 \mathrm{~b}$ shows voltage oscillations $V\left(I_{\text {mod }}\right)$ for different values of fixed bias current close to $I_{c, \text { max }}$. From the oscillation period (current $I_{m o d, 0}$ required to couple one flux quantum $\Phi_{0}=$ $\mathrm{h} / 2 \mathrm{e} \approx 2.0678 \times 10^{-15} \mathrm{Vs}$ to the SQUID) we determine the inverse mutual inductance $\mathrm{M}_{\mathrm{f}}^{-1}=I_{\text {mod, }} / \Phi_{0}$ between the modulation line and the SQUID, which ranges from about 2 $\mathrm{mA} / \Phi_{0}$ (SQ-1) to $2.8 \mathrm{~mA} / \Phi_{0}$ (SQ-2) for the two exemplary SQUIDs. We note that $\mathrm{M}_{\mathrm{f}}{ }^{-1}$ for nSQ-1 is larger than for SQ-2, which may be due to flux focusing, caused by the larger washer of SQ-1 [45]. For SQ-2, the small shift $\Delta I_{\text {mod }}= \pm 83 \mu \mathrm{A}$ of the maxima in $I_{c}\left(I_{\text {mod }}\right)$ for opposite polarity (c.f. Figure 13a) is, as expected, very close to the symmetric single JJ critical current $I_{c, \max } / 2=86.3 \mu \mathrm{A}$. This means that the asymmetry in the $I_{c}\left(I_{\text {mod }}\right)$ oscillations is likely dominated by inductance asymmetry due to asymmetric current biasing of the SQUIDs, and that an asymmetry in the critical currents of the two JJs is negligible.

For negligible noise rounding and negligible critical current asymmetry, we can then estimate the screening parameter

$$
\beta_{L}=I_{c, \max } L_{S Q} / \Phi_{0}
$$


$\beta_{L}$ is derived using the relation between the normalized modulation depth $\Delta I_{c} / I_{c, \max }$ vs. $\beta_{L}$ (with $\Delta I_{c}=I_{c, \max }-I_{c, \min }$ ), which has been obtained from numerical simulations based on the RCSJ model [1]. From Equation (3), we then obtain $L_{S Q} \approx 1.4 \mathrm{pH}$ (SQ-1) resp. $1.7 \mathrm{pH}$ (SQ-2). The maximum modulation voltage of our exemplary SQUIDs is $71 \mu \mathrm{V}$ for SQ-1 and $61 \mu \mathrm{V}$ for SQ-2 (c.f. Figure 13b) and the derived maximum transfer coefficients are $V_{\Phi}=944 \mu \mathrm{V} / \Phi_{0}$ (SQ-1) and $441 \mu \mathrm{V} / \Phi_{0}$ (SQ-2). All characteristic parameters for both SQUIDs are summarized in Table 3 together with results from ref. [42], where nominally the same layout was fabricated, but with a two-Nb-layer fabrication process. The deviations from our results (regarding the mutual inductance, for example) can be explained, since the SQUIDs were fabricated with another deposition system using nominally an identical HfTi target. However, in general, the SQUID characteristics of the SQUIDs which were investigated for this paper are in accordance with the parameters from ref. [42].

The critical current densities of both investigated SQUIDs stemming from different wafers meet the expectations regarding data from former measurements of critical current densities of single JJs [32,33] (fabricated with the process for only two independent $\mathrm{Nb}$ layers), including different wafers with varying thickness of the HfTi barrier of the JJs. In addition to 16 SQUIDs of the types SQ-1 and SQ-2 from wafer 1 and wafer 2, where we demonstrated a high yield for JJ vias, a total of eight single JJs from the same two wafers with a HfTi barrier thickness of $20 \mathrm{~nm}$ or $21 \mathrm{~nm}$ were investigated. For 10 SQUIDs with $d_{H f T i}=20 \mathrm{~nm}$, the critical current density was $j_{c}=(309 \pm 57) \mathrm{kA} / \mathrm{cm}^{2}$ and $j_{c}=(243 \pm 46) \mathrm{kA} / \mathrm{cm}^{2}$ for 6 SQUIDs with $d_{H f T i}=21 \mathrm{~nm}$. The critical current density of five single JJs was $(261 \pm 41) \mathrm{kA} / \mathrm{cm}^{2}$ for the thinner barrier and $(170 \pm 10) \mathrm{kA} / \mathrm{cm}^{2}$ for three JJs with the thicker barrier.

The mean value of $j_{\mathrm{c}}\left(d_{\mathrm{HfTi}}\right)$ for all SQUIDs and for single JJs from wafer 1 and wafer 2 measured here and the critical current density of single JJs with the same nominal width, but fabricated with the process for two independent $\mathrm{Nb}$ layers and with different HfTi barrier thicknesses, are shown in Figure 14. The mean values of the SQUIDs and single JJs, which were prepared with the extended fabrication process, agree reasonably well with the data of the single JJs, which were fabricated with the two $\mathrm{Nb}$ layer process.

Additionally, data of single JJs with nominal widths of $200 \mathrm{~nm}$ from [33] are shown for comparison. For those, $j_{c}$ is calculated using the effective area (considering deviations from the nominal area due to the patterning process and due to edge damage).

For our JJs, the expression [46]

$$
j_{c}=j_{c 0} \cdot \exp \left[-d_{H f T i} / \xi_{N}\right]
$$

can be used [33] to describe the dependence of the critical current density on the HfTi barrier thickness, where $\xi_{\mathrm{N}}$ is the coherence length in the normal conducting barrier. In ref. [33], the fitting parameters are $j_{c 0}=(27 \pm 13) \mathrm{MA} / \mathrm{cm}^{2}$ and $\xi_{N}=(4.85 \pm 0.75) \mathrm{nm}$. In general, the fit function agrees well with our measurement results for SQUIDs and single JJs. Since our data were determined using the designed area, which is somewhat larger than the effective area of the JJs, our data are slightly below the curve from ref. [33]. 


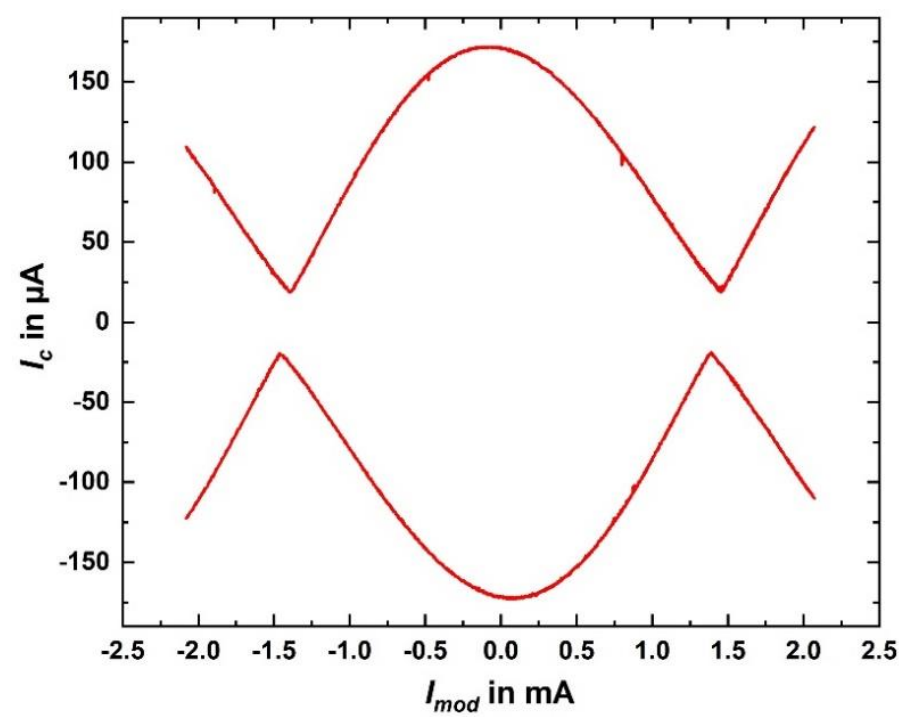

(a)

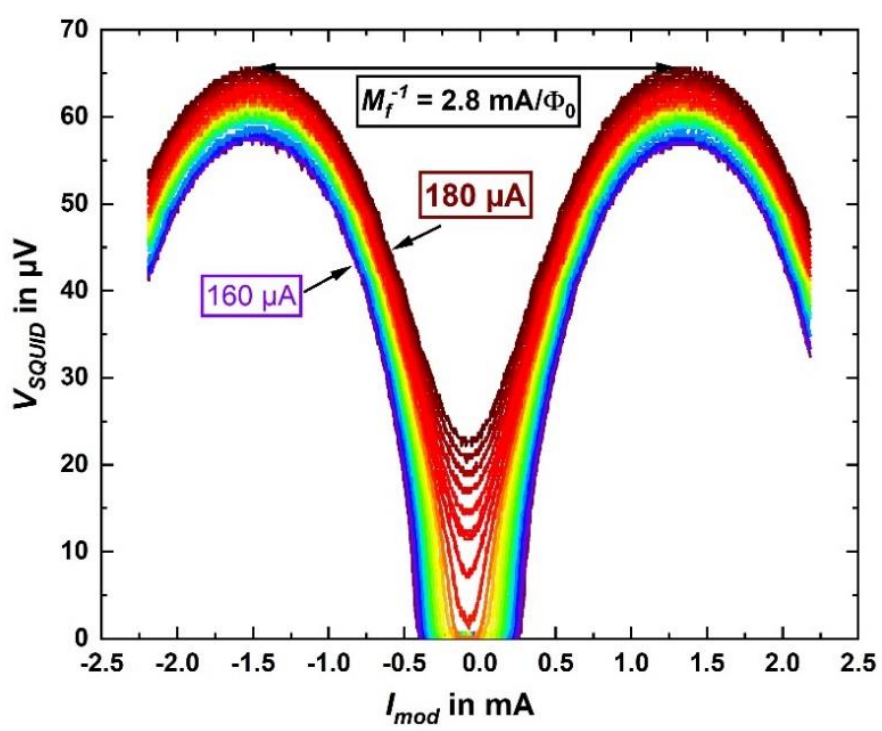

(b)

Figure 13. SQUID oscillations of SQ-2 at $4.2 \mathrm{~K}$. (a) Critical current vs. modulation current. (b) Voltage vs. modulation current for constant bias currents (from 160 to $180 \mu \mathrm{A}$, in $1 \mu \mathrm{A}$ steps).

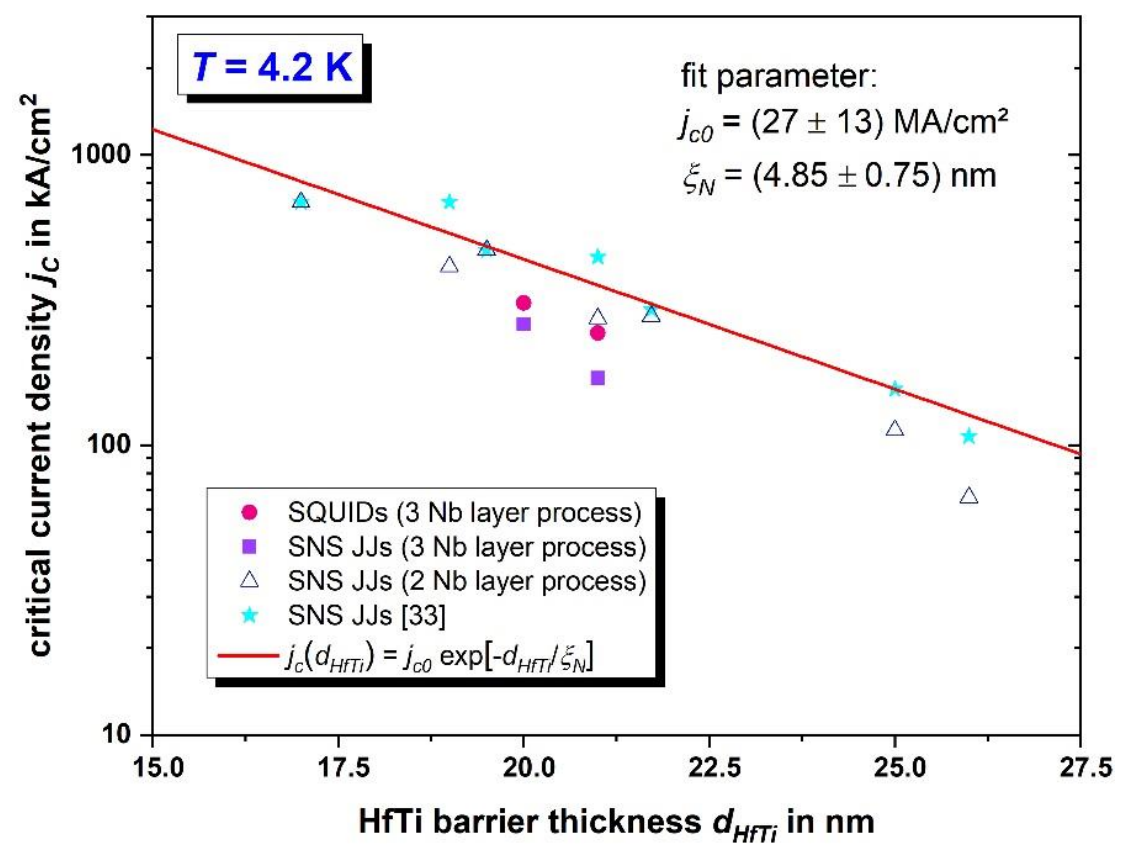

Figure 14. Critical current densities at $4.2 \mathrm{~K}$ vs. HfTi barrier thickness of SQUID types SQ-1 and SQ-2 and single HfTi SNS-JJs, all with nominal JJ width of $200 \mathrm{~nm}$.

Table 3. Characteristic parameters of two representative SQUIDs and a SQUID from ref. [42] with the same layout for comparison.

\begin{tabular}{|c|c|c|c|c|c|c|c|c|c|c|}
\hline & $\begin{array}{l}d_{H f T i} \\
(\mathrm{~nm})\end{array}$ & $\begin{array}{l}I_{c, \min } \\
(\mu \mathrm{A})\end{array}$ & $\begin{array}{c}I_{c, \max } \\
(\mu \mathrm{A})\end{array}$ & $\begin{array}{c}j_{c} \\
\left(\mathrm{kA} / \mathrm{cm}^{2}\right)\end{array}$ & $\begin{array}{c}R_{S Q} \\
(\mathrm{~m} \Omega)\end{array}$ & $\begin{array}{c}V_{c} \\
(\mu \mathrm{V})\end{array}$ & $\begin{array}{c}V_{\Phi} \\
\left(\mu \mathrm{V} / \Phi_{0}\right)\end{array}$ & $\begin{array}{c}1 / M_{\mathrm{f}} \\
\left(\mathrm{mA} / \Phi_{0}\right)\end{array}$ & $\beta_{L}$ & $\begin{array}{l}L_{\mathrm{SQ}} \\
(\mathrm{pH})\end{array}$ \\
\hline SQ-1 & 20 & 30.3 & 238.3 & 298 & 343 & 81.7 & 944 & 2.0 & 0.16 & 1.4 \\
\hline SQ-2 & 21 & 19.2 & 172.6 & 216 & 415 & 71.6 & 441 & 2.8 & 0.14 & 1.7 \\
\hline ref. [42] & 24 & 21 & 178 & 233 & 233 & 41.5 & 100 & 4.4 & 0.18 & 2.1 \\
\hline
\end{tabular}




\section{Summary and Outlook}

We extended our fabrication technology for nanoSQUIDs with SNS JJs from originally two to three independent layers of $\mathrm{Nb}$. Thereby, six test wafers were fabricated to optimize the process and to assess the high yield and characteristics of capacitor structures and vias as well as single Josephson junctions and dc SQUIDs.

The circuits on all six wafers showed a high yield of investigated parallel-plate capacitors having different sizes and plates in all possible combinations of $\mathrm{Nb}$ layers. Measurements of the capacitance of those capacitors were performed to deduce the height of the dielectric $\mathrm{SiO}_{2}$ layer between the capacitors' plates. The deviation from of the designed values can be explained by the inhomogeneity of the CMP process, causing a difference in height of $\mathrm{SiO}_{2}$ across the wafers. Fabricating high aspect ratio IDCs was challenging due to the large area of the structures which must be fabricated without any defects, causing a reduced yield; still we demonstrate the possibility to obtain the capacitances very precisely in agreement with the capacitor design. Furthermore, we studied the yield of $\mathrm{Nb}$ vias and $\mathrm{JJ}$ vias connecting the three independent layers of $\mathrm{Nb}$, which was very high for all investigated $\mathrm{Nb}$ vias. For JJ vias, which were fabricated using a CMP process, enabling additionally much smaller dimensions, the yield suffered much more from inter-wafer inhomogeneity (polishing grade).

To demonstrate the suitability of our new fabrication process for the realization of SQUIDs, we fabricated dc SQUID gradiometer within three fabrication runs for two wafers, respectively, and investigated the JJ and SQUID characteristics. Since all investigated SQUIDs showed reasonable critical currents and modulation upon applying a modulation current, we conclude a very high yield for the first fabrication run. The two subsequent fabrication runs showed an exceedingly lower yield due to a too strong or too weak polishing, indicating that the CMP fabrication step is very crucial to achieve a high yield for nanoSQUIDs. Still, the deviation of the critical current density from the designed values for all investigated SQUIDs and single JJs from the first fabrication run was small. Therefore, we conclude that the performance of SQUIDs and single JJs is not affected by the extension of the fabrication process from originally two to three independent layers of $\mathrm{Nb}$, since they show IVCs which fully match our expectations regarding their HfTi barrier thicknesses. In addition, the suitability of our fabrication process for different types of precise on-chip capacitors, which can be used as auxiliary components for more complex SQUID circuits, could be demonstrated.

The $\mathrm{Nb}$ multilayer technology presented in this paper offers the possibility to increase the density of structures on one wafer to create more complex and advanced nanoSQUID circuits, e.g., three-dimensional vector nanoSQUIDs [28] and nanoSQUID susceptometers [29].

In future work we expect to improve upon the yield by enhancing the controllability of the CMP process by introducing additional supporting structures as well as by adapting the etching times to optimize the steepness of the structures' sidewalls. Furthermore, we will optimize the design of the dc SQUID gradiometers to increase the coupling to magnetic nanoparticles, and we will perform detailed studies of nanoSQUID noise properties.

Author Contributions: Conceptualization, S.B., R.K., J.B., D.K. and O.K.; methodology, S.W., J.A., T.W., S.B., R.K., J.B., D.K. and O.K.; software, O.K.; validation, S.W. and J.L.; investigation, S.W., J.L., J.A., T.W. and O.K.; resources, T.W.; writing-original draft preparation, S.W., R.K., J.B., D.K. and O.K.; writing—review and editing, J.L., S.B., R.K., J.B., D.K. and O.K.; visualization, D.K. and O.K.; supervision, D.K. and O.K.; project administration, S.B., R.K. and D.K.; funding acquisition, S.B., R.K., D.K. and O.K. All authors have read and agreed to the published version of the manuscript.

Funding: This research was funded by the Deutsche Forschungsgemeinschaft (DFG) (KI 698/3-2, KO 1303/13-2, BE 6680/1-2) and by the COST action NANOCOHYBRI (CA16218).

Institutional Review Board Statement: Not applicable.

Informed Consent Statement: Not applicable. 
Data Availability Statement: The data presented in this study are available on request from the corresponding author. The data are not publicly available because there is no public cloud memory available at the federal institute of PTB.

Acknowledgments: The authors thank K. Störr, M. Petrich, R. Wendisch, R. Gerdau, P. Hinze, J. Felgner and K. Wurster for their help throughout the wafer fabrication, support at the SEM equipment and sample characterization.

Conflicts of Interest: The authors declare no conflict of interest.

\section{References and Note}

1. Clarke, J.; Braginski, A.I. (Eds.) The SQUID Handbook Vol.I Fundamentals and Technology of SQUIDs and SQUID Systems; Wiley-VCH: Weinheim, Germany, 2004.

2. Clarke, J.; Braginski, A.I. (Eds.) The SQUID Handbook Vol.II Applications of SQUIDs and SQUID Systems; Wiley-VCH: Weinheim, Germany, 2006.

3. Granata, C.; Vettoliere, A. Nano superconducting quantum interference devices: A powerful tool for nanoscale investigations. Phys. Rep. 2016, 614, 1-69. [CrossRef]

4. Martínez-Pérez, M.J.; Koelle, D. NanoSQUIDs: Basics \& recent advances. Phys. Sci. Rev. 2017, $2,20175001$.

5. Kirtley, J.R.; Ketchen, M.B.; Staviasz, K.G.; Sun, J.Z.; Gallagher, W.J.; Blanton, S.H.; Wind, S.J. High-resolution scanning SQUID microscope. Appl. Phys. Lett. 1995, 66, 1138-1140. [CrossRef]

6. Kirtley, J.R. Prospects for imaging magnetic nanoparticles using a scanning SQUID microscope. Supercond. Sci. Technol. 2009, 22, 064008. [CrossRef]

7. Vasyukov, D.; Anahory, Y.; Embon, L.; Halbertal, D.; Cuppens, J.; Neeman, L.; Finkler, A.; Segev, Y.; Myasoedov, Y.; Rappaport, M.L.; et al. A scanning superconducting quantum interference device with single electron spin sensitivity. Nat. Nanotechnol. 2013, 8, 639-644. [CrossRef]

8. Hazra, D.; Kirtley, J.R.; Hasselbach, K. Nano-superconducting quantum interference devices with continuous read out at millikelvin temperatures. Appl. Phys. Lett. 2013, 103, 093109. [CrossRef]

9. Kirtley, J.R.; Paulius, L.; Rosenberg, A.J.; Palmstrom, J.C.; Holland, C.M.; Spanton, E.M.; Schiessl, D.; Jermain, C.L.; Gibbons, J.; Fung, Y.-K.-K.; et al. Scanning SQUID susceptometers with sub-micron spatial resolution. Rev. Sci. Instrum. 2016, 87, 093702. [CrossRef]

10. Reith, P.; Wang, X.R.; Hilgenkamp, H. Analysing magnetism using scanning SQUID microscopy. Rev. Sci. Instrum. 2017, 88, 123706. [CrossRef]

11. Wyss, M.; Gliga, S.; Vasyukov, D.; Ceccarelli, L.; Romagnoli, G.; Cui, J.; Kleibert, A.; Stamps, R.L.; Poggio, M. Stray-field imaging of a chiral artificial spin ice during magnetization reversal. ACS Nano 2019, 13, 13910-13916. [CrossRef]

12. Anahory, Y.; Naren, H.R.; Lachman, E.O.; Sinai, S.B.; Uri, A.; Embon, L.; Yaakobi, E.; Myasoedov, Y.; Huber, M.E.; Klajn, R.; et al. SQUID-on-tip with single-electron spin sensitivity for high-field and ultra-low temperature nanomagnetic imaging. Nanoscale 2020, 12, 3174-3182. [CrossRef]

13. Uri, A.; Kim, Y.; Bagani, K.; Lewandowski, C.K.; Grover, S.; Auerbach, N.; Lachman, E.O.; Myasoedov, Y.; Taniguchi, T.; Watanabe, K.; et al. Nanoscale imaging of equilibrium quantum Hall edge currents and of the magnetic monopole response in graphene. Nat. Phys. 2020, 16, 164-170. [CrossRef]

14. Wernsdorfer, W. Classical and quantum magnetization reversal studied in nanometersized particles and clusters. Adv. Chem. Phys. 2001, 118, 99-190.

15. Jamet, M.; Wernsdorfer, W.; Thirion, C.; Mailly, D.; Dupuis, V.; Mélinon, P.; Pérez, A. Magnetic anisotropy of a single cobalt nanocluster. Phys. Rev. Lett. 2001, 86, 4676-4679. [CrossRef] [PubMed]

16. Wernsdorfer, W. From micro- to nano-SQUIDs: Applications to nanomagnetism. Supercond. Sci. Technol. 2009, $22,064013$. [CrossRef]

17. Hao, L.; Aßmann, C.; Gallop, J.C.; Cox, D.; Ruede, F.; Kazakova, O.; Josephs-Franks, P.; Drung, D.; Schurig, T. Detection of single magnetic nanobead with a nano-superconducting quantum interference device. Appl. Phys. Lett. 2011, 98, 092504. [CrossRef]

18. Buchter, A.; Nagel, J.; Rüffer, D.; Xue, F.; Weber, D.P.; Kieler, O.F.; Weimann, T.; Kohlmann, J.; Zorin, A.B.; Russo-Averchi, E.; et al Reversal mechanism of an individual Ni nanotube simultaneously studied by torque and SQUID magnetometry. Phys. Rev. Lett. 2013, 111, 067202. [CrossRef]

19. Schwarz, T.; Wölbing, R.; Reiche, C.F.; Müller, B.; Martínez-Pérez, M.J.; Mühl, T.; Büchner, B.; Kleiner, R.; Koelle, D. Low-noise $\mathrm{YBa}_{2} \mathrm{Cu}_{3} \mathrm{O}_{7}$ nano-SQUIDs for performing magnetization-reversal measurements on magnetic nanoparticles. Phys. Rev. Appl. 2015, 3, 044011. [CrossRef]

20. Martínez-Pérez, M.J.; Müller, B.; Schwebius, D.; Korinski, D.; Kleiner, R.; Sesé, J.; Koelle, D. NanoSQUID magnetometry of individual cobalt nanoparticles grown by focused electron beam induced deposition. Supercond. Sci. Technol. 2017, $30,024003$. [CrossRef]

21. Martínez-Pérez, M.J.; Pablo-Navarro, J.; Müller, B.; Stankievicz, J.; Kleiner, R.; Magén, C.; Koelle, D.; de Teresa, J.M.; Sesé, J. NanoSQUID magnetometry on individual as-grown and annealed Co nanowires at variable temperature. Nano Lett. 2018, 18, 7674-7682. [CrossRef] 
22. Martínez-Pérez, M.J.; Müller, B.; Lin, J.; Rodriguez, L.A.; Snoeck, E.; Kleiner, R.; Sesé, J.; Koelle, D. Magnetic vortex nucleation and annihilation in bi-stable ultrasmall ferromagnetic particles. Nanoscale 2020, 12, 2587-2595. [CrossRef]

23. Schwarz, T.; Nagel, J.; Wölbing, R.; Kemmler, M.; Kleiner, R.; Koelle, D. Low-noise nano superconducting quantum interference device operating in tesla magnetic fields. ACS Nano 2013, 7, 844-850. [CrossRef] [PubMed]

24. Wölbing, R.; Nagel, J.; Schwarz, T.; Kieler, O.; Weimann, T.; Kohlmann, J.; Zorin, A.B.; Kemmler, M.; Kleiner, R.; Koelle, D. Nb nano superconducting quantum interference devices with high spin sensitivity for operation in magnetic fields up to $0.5 \mathrm{~T}$. Appl. Phys. Lett. 2013, 102, 192601. [CrossRef]

25. Bouchiat, V. Detection of magnetic moments using a nano-SQUID: Limits of resolution and sensitivity in near-field SQUID magnetometry. Supercond. Sci. Technol. 2009, 22, 064002. [CrossRef]

26. Nagel, J.; Konovalenko, K.B.; Kemmler, M.; Turad, M.; Werner, R.; Kleisz, E.; Menzel, S.; Klingeler, R.; Büchner, B.; Kleiner, R.; et al. Resistively shunted $\mathrm{YBa}_{2} \mathrm{Cu}_{3} \mathrm{O}_{7}$ grain boundary junctions and low-noise SQUIDs patterned by a focused ion beam down to $80 \mathrm{~nm}$ linewidth. Supercond. Sci. Technol. 2011, 24, 015015. [CrossRef]

27. Wölbing, R.; Schwarz, T.; Müller, B.; Nagel, J.; Kemmler, M.; Kleiner, R.; Koelle, D. Optimizing the spin sensitivity of grain boundary junction nanoSQUIDs—-towards detection of small spin systems with single-spin resolution. Supercond. Sci. Technol. 2014, 27, 125007. [CrossRef]

28. Martínez-Pérez, M.J.; Gella, D.; Müller, B.; Morosh, V.; Wölbing, R.; Sesé, J.; Kieler, O.; Kleiner, R.; Koelle, D. Three-Axis Vector Nano Superconducting Quantum Interference Device. ACS Nano 2016, 10, 8308-8315. [CrossRef] [PubMed]

29. Bechstein, S.; Köhn, C.; Drung, D.; Storm, J.-H.; Kieler, O.; Morosh, V.; Schurig, T. Investigation of nanoSQUID designs for practical applications. Supercond. Sci. Technol. 2017, 30, 034007. [CrossRef]

30. Hagedorn, D.; Kieler, O.; Dolata, R.; Behr, R.; Müller, F.; Kohlmann, J.; Niemeyer, J. Modified fabrication of planar sub- $\mu m$ superconductor-normal metal-superconductor Josephson junctions for use in a Josephson arbitrary waveform synthesizer. Supercond. Sci. Technol. 2006, 19, 294-298. [CrossRef]

31. Kieler, O.F.; Iuzzolino, R.; Kohlmann, J. Sub- $\mu$ m SNS Josephson junction arrays for the Josephson arbitrary waveform synthesizer. IEEE Trans. Appl. Supercond. 2009, 19, 230-233. [CrossRef]

32. Kieler, O. Pulsgetriebenes AC-Josephson-Spannungsnormal-Josephson Arbitrary Waveform Synthesizer. Ph.D. Thesis, TU Ilmenau, Physikalisch-Technische Bundesanstalt, Braunschweig, Germany, 2017. PTB-Bericht E-109.

33. Morosh, V.; Linek, J.; Müller, B.; Martínez-Pérez, M.J.; Wolter, S.; Weimann, T.; Beyer, J.; Schurig, T.; Kieler, O.; Zorin, A.B.; et al. Transport and noise properties of sub-100-nm planar Nb Josephson junctions with metallic HfTi barrier for nanoSQUID applications. Phys. Rev. Appl. 2020, 14, 054072. [CrossRef]

34. Tolpygo, S.K.; Bokhovsky, V.; Rastogi, R.; Zarr, S.; Day, A.L.; Golden, E.; Weir, T.J.; Wynn, A.; Johnson, L.M. Advanced Fabrication Processes for Superconductor Electronics: Current Status and New Developments. IEEE Trans. Appl. Supercond. 2019, $29,1102513$. [CrossRef]

35. Olaya, D.; Castellanos-Beltran, M.; Pulecio, J.; Biesecker, J.; Khadem, S.; Lewitt, T.; Hopkins, P. Planarized Process for Single-FluxQuantum Circuits With Self-Shunted $\mathrm{Nb} / \mathrm{Nb}_{\mathrm{x}} \mathrm{Si}_{1-\mathrm{x}} / \mathrm{Nb}$ Josephson Junctions. IEEE Trans. Appl. Supercond. 2019, $29,1101708$. [CrossRef]

36. Nagasawa, S.; Hinode, K.; Satoh, T.; Hidaka, M.; Akaike, H.; Fujimaki, A.; Yoshikawa, N.; Takagi, K.; Takagi, N. Nb 9-Layer Fabrication Process for Superconducting Large-Scale SFQ Circuits and Its Process Evaluation. IEICE Trans. Electron. 2014, 97, 132-140. [CrossRef]

37. Tolpygo, S.K.; Bolkhovsky, V.; Weir, T.; Johnson, L.M.; Oliver, W.D.; Gouker, M.A. Deep sub-micron stud-via technology for superconductor VLSI circuits. J. Phys. Conf. Ser. 2013, 507, 042043. [CrossRef]

38. The commercial instruments are identified in this paper only in order to adequately specify the experimental procedure. Such identification does not imply recommendation or endorsement by the PTB.

39. Felgner, J.; Kieler, O.F.; Schurr, J.; Herick, J.; Bauer, S.; Wendisch, R.; Störr, K. Evaluation of Material Parameters for on-Chip Capacitors and Resistors for Superconducting Circuits; Tagung Kryoelektronische Bauelemente: Bad Aibling, Germany, 2017.

40. Naini, A.; Green, M. Fringing fields in a parallel-plate capacitor. Am. J. Phys. 1977, 45, 877. [CrossRef]

41. Hong, J.-S.; Lancaster, M.J. Microstrip Filters for RF/Microwave Applications; John Wiley \& Sons, Inc.: Hoboken, NJ, USA, 2001.

42. Nagel, J.; Kieler, O.F.; Weimann, T.; Wölbing, R.; Kohlmann, J.; Zorin, A.B.; Kleiner, R.; Koelle, D.; Kemmler, M. Superconducting quantum interference devices with submicron $\mathrm{Nb} / \mathrm{HfTi} / \mathrm{Nb}$ junctions for investigation of small magnetic particles. Appl. Phys. Lett. 2011, 99, 032506. [CrossRef]

43. Stewart, W.C. Current-voltage characteristics of Josephson junctions. J. Appl. Phys. Lett. 1968, 12, 277-280. [CrossRef]

44. McCumber, D.E. Effect of ac impedance on dc voltage-current characteristics of Josephson junctions. J. Appl. Phys. 1968, 39, 3113-3118. [CrossRef]

45. Ketchen, M.B.; Gallagher, W.J.; Kleinsasser, A.W.; Murphy, S.; Clem, J.R. DC SQUID flux focuser. 3. International Conference on Superconducting Quantum Devices; Walter de Gruyter: Berlin, Germany, 1985.

46. Delin, K.A.; Kleinsasser, A.W. Stationary properties of high-critical-temperature proximity effect Josephson junctions. Supercond. Sci. Technol. 1996, 9, 227-269. [CrossRef] 